\title{
A Theoretical Limit for the ML Performance of MIMO Systems based on Lattices
}

\author{
Koralia N. Pappi, Vasileios M. Kapinas, and George K. Karagiannidis \\ Department of Electrical \& Computer Engineering, Aristotle University of Thessaloniki, Thessaloniki, Greece, \\ E-mails: \{kpappi, kapinas, geokarag\}@ auth.gr
}

\begin{abstract}
We employ the theory of multidimensional lattice constellations in order to evaluate the maximum-likelihood (ML) performance of a general multiple-input multiple-output MIMO system. We show that the lattice representation of the equivalent system model enables a mapping of the received signal set to a single point in a multidimensional constellation, with the generator matrix being equal to the equivalent channel matrix. With the aid of this geometrical interpretation, we derive a performance limit for a general MIMO system in terms of the symbol error probability, which can be written in closed form for various designs. Application of the proposed analysis to popular MIMO schemes reveals the tractability of the approach and validates that designs exploiting the available degrees of freedom in a better way approach the proposed performance limit.
\end{abstract}

\section{INTRODUCTION}

Multiple-input multiple-output (MIMO) techniques have long now been an efficient means of providing extra degrees of freedom (DoF) in the wireless link, that can be exploited towards more reliable communication and/or faster data transfer. To reach these goals, numerous multiple-antenna transceiver designs have been proposed so far to offer versatile benefits in terms of signal-to-noise ratio (SNR) improvement and capacity increase, such as diversity, array and multiplexing gains [1]. Nevertheless, the success of these systems depends heavily on the ability of the receiver to achieve the maximum-likelihood (ML) performance in the detection process. Since this task is sometimes very hard due to complexity issues induced in the MIMO decoder [2], several papers in the literature are devoted in the study and performance analysis of MIMO systems with sub-optimum [3] or near-optimum receivers [4].

For a MIMO system with given DoF, it would always be desirable to have a theoretical measure of its optimum performance, whether being approachable or not in practical scenarios. To this end, various ML performance evaluation studies have been conducted by researchers targeting specific MIMO designs, such as orthogonal space-time block codes (STBC) [5], quasi-orthogonal STBC [6] and closedloop MIMO systems [7]. In all these cases, the partial/full orthogonality of the block code and the channel state information (CSI) availability at the transmitter side reduce or

This research has been co-financed by the European Union (European Social Fund - ESF) and Greek national funds through the Operational Program "Education and Lifelong Learning" of the National Strategic Reference Framework (NSRF) - Research Funding Program: THALES: Reinforcement of the interdisciplinary and/or inter-institutional research and innovation. even vanish the self interference terms at the matched filter output, thus relaxing the decoder complexity and rendering the performance analysis more straightforward. However, for more arbitrary multiple-antenna transmission strategies, the conventional tractable analytical tools give way to more intuitive approaches, such as [8] on space-time coding and [9] on general MIMO systems.

In this paper, we establish a novel framework for the ML performance evaluation of an arbitrary MIMO system, using the analysis introduced in [10] for the symbol error probability (SEP) calculation of multidimensional signal sets perturbed by additive white Gaussian noise (AWGN). In more detail, we first build upon these results to evaluate the SEP of a $2 N$ dimensional lattice, whose generator matrix is equal to the $2 N \times 2 N$ equivalent real MIMO channel matrix, ${ }^{1}$ distorted by AWGN. Based on this geometrical interpretation, in Corollary 1 we obtain a theoretical performance limit for all MIMO systems that can be represented by the particular equivalent set of $2 N$ equations. This implies that, even if all available MIMO DoF are efficiently exploited, the optimum ML performance cannot exceed this limit, which is derived here in closed form for the case of Square Quadrature Amplitude Modulation (SQAM) and Rayleigh flat fading channel. The derived limits could be utilized as benchmarks for the efficient design of more sophisticated MIMO setups, such as systems employing transmission using rotated constellations and feedback-based systems, among others.

\section{MIMO SYSTEM MODEL}

We consider a MIMO system, which, after simple signal processing at the receiver, can be modeled by an $N \times N$ complex-valued system of equations. This representation is usually called the equivalent system model and is given by

$$
\mathbf{y}=\mathbf{H x}+\mathbf{w}
$$

where $\mathbf{y} \in \mathbb{C}^{N}$ is the received signal vector, $\mathbf{H} \in \mathbb{C}^{N \times N}$ is the channel matrix, $\mathbf{x} \in \mathbb{C}^{N}$ is the transmitted signal vector - hereafter denoted as supersymbol - and whose entries are independently drawn from an SQAM constellation and $\mathbf{w} \in$ $\mathbb{C}^{N}$ is the complex additive white Gaussian noise (AWGN)

\footnotetext{
${ }^{1}$ This does not necessarily imply the same number of transmit and receive antennas, since the size of the equivalent set of equations depends also on the number of time-slots and the number of information symbols per block [11].
} 
vector with zero mean and covariance matrix $\sigma_{c}^{2} \mathbf{I}_{N}$, where $I_{N}$ is the identity matrix of order $N$.

\section{A. Real-valued Representation of the Received Signal Set}

The $N$-dimensional complex-valued problem in (1) can be decomposed into a $2 N$-dimensional real-valued problem, which then can be written as

$$
\tilde{\mathbf{y}}=\tilde{\mathbf{H}} \tilde{\mathbf{x}}+\tilde{\mathbf{w}}
$$

where

$$
\begin{aligned}
& \tilde{\mathbf{y}}=\left[\begin{array}{l}
\Re(\mathbf{y}) \\
\Im(\mathbf{y})
\end{array}\right], \quad \tilde{\mathbf{H}}=\left[\begin{array}{cc}
\Re(\mathbf{H}) & -\Im(\mathbf{H}) \\
\Im(\mathbf{H}) & \Re(\mathbf{H})
\end{array}\right], \\
& \tilde{\mathbf{x}}=\left[\begin{array}{l}
\Re(\mathbf{x}) \\
\Im(\mathbf{x})
\end{array}\right], \quad \tilde{\mathbf{w}}=\left[\begin{array}{c}
\Re(\mathbf{w}) \\
\Im(\mathbf{w})
\end{array}\right],
\end{aligned}
$$

and $\Re(\cdot)$ and $\Im(\cdot)$ denote the real and imaginary part of a complex vector or matrix, respectively.

In (2), $\tilde{\mathbf{w}} \in \mathbb{R}^{2 N}$ is the AWGN vector with zero mean and covariance matrix $\sigma^{2} \mathbf{I}_{2 N}$, where $\sigma^{2}=\frac{\sigma_{c}^{2}}{2}$. For a given channel realization, the channel transition probabilities are given by

$$
p(\tilde{\mathbf{y}} \mid \tilde{\mathbf{x}}, \tilde{\mathbf{H}})=\left(2 \pi \sigma^{2}\right)^{-N} \exp \left(-\frac{1}{2 \sigma^{2}}\|\tilde{\mathbf{y}}-\tilde{\mathbf{H}} \tilde{\mathbf{x}}\|^{2}\right) .
$$

\section{B. A Useful Lattice Representation of the System Model}

The transmitted supersymbol $\mathbf{x}$ whose entries are drawn from an SQAM constellation can be considered as a lattice point of a $2 N$-dimensional real lattice. Specifically, its real representation $\tilde{\mathbf{x}}$ can be written as

$$
\tilde{\mathbf{x}}=\mathbf{M u}+\mathbf{x}_{0},
$$

where $\mathbf{M}=a \mathbf{I}_{2 N}, a \in \mathbb{R}^{+}$is the generator matrix of the lattice which is a scaled version of the identity matrix, $\mathbf{x}_{0} \in \mathbb{R}^{2 N}$ is an offset vector which minimizes the mean constellation energy and

$$
\mathbf{u}=\left[u_{1} u_{2} \ldots u_{2 N}\right]^{T}, \quad u_{i} \in\{0,1, \ldots, \sqrt{M}-1\},
$$

where $M$ denotes the size of the $M$-SQAM constellation in use. In order to examine various SQAM constellations with different size $M$, we set $|\operatorname{det}(\mathbf{M})|=1$, and thus $\mathbf{M}=\mathbf{I}_{2 N}$, while the minimum distance between signal points is set to $d_{\min }=1$ as in [12]. Subsequently, we define the Volume to Noise Ratio (VNR) as $\rho=\frac{1}{\sigma^{2}}$. In the following, the offset vector $\mathbf{x}_{0}$ is omitted, since it does not affect our analysis.

Substituting (5) in (2), the received real vector yields

$$
\tilde{\mathbf{y}}=\tilde{\mathbf{H}} \mathbf{M u}+\tilde{\mathbf{w}}=\tilde{\mathbf{H}} \mathbf{u}+\tilde{\mathbf{w}}
$$

which can also be considered as a point on a constellation, carved from a lattice with generator matrix $\mathbf{M}^{\prime}=\tilde{\mathbf{H}}$, distorted by AWGN.

\section{ERROR PERFORMANCE EVALUATION}

\section{A. Supersymbol and Symbol Error Probability}

The received vector in (7) for a specific realization of the matrix $\tilde{\mathbf{H}}$ is considered as a lattice point on a finite lattice constellation. It's shaping region is a parallelotope, formed by a vector basis defined by $\tilde{\mathbf{H}}$, where each column represents a basis vector. In fact, from (5) and (7) the constellation can be considered as $2 N$-dimensional, with $\sqrt{M}$ points along each basis vector. Let us now define the sets [10]

$$
\mathcal{S}_{k, p} \subseteq \mathcal{S}_{2 N, 1}=\left\{\tilde{\mathbf{h}}_{1}, \tilde{\mathbf{h}}_{2}, \ldots, \tilde{\mathbf{h}}_{2 N}\right\},
$$

where $\tilde{\mathbf{h}}_{i}, i=1,2, \ldots, 2 N$, denotes the $i$ th column of the matrix $\tilde{\mathbf{H}}$ which is a basis vector of the parallelotope shaping region as well as the lattice, $k$ denotes the number of vectors which an $\mathcal{S}_{k, p}$ subset contains with $0<k \leq 2 N$, and $p$ is an enumeration index with $p=1,2, \ldots,\left(\begin{array}{c}2 N \\ k\end{array}\right)$, which denotes the different $\mathcal{S}_{k, p}$ subsets. We finally denote the $k$-dimensional Voronoi region of a sublattice formed by the basis vectors in $\mathcal{S}_{k, p}$ by $\mathcal{V}_{\mathcal{S}_{k, p}}$.

According to [10], for a given channel realization, the error probability of the multidimensional signal vector, which will be called supersymbol error probability (SSEP), satisfies

$$
P_{s s}(\rho \mid \tilde{\mathbf{H}})=1-\frac{\sum_{k=0}^{2 N}(\sqrt{M}-1)^{k} \sum_{p=1}^{\left(\begin{array}{c}
2 N \\
k
\end{array}\right)} J_{k, p}}{M^{N}},
$$

where the integrals $J_{k, p}$ are given by

$$
J_{k, p}=\int_{\mathcal{V}_{\mathcal{S}_{k, p}}} p\left(\mathbf{z}_{k}\right) \mathrm{d} \mathbf{z}_{k}, \quad 0<k \leq 2 N,
$$

where $p\left(\mathbf{z}_{k}\right)$ is the $k$-dimensional pdf of the AWGN, while $J_{0,1} \triangleq 1$. Subsequently, the SSEP averaged on all channel realizations is given by

$$
P_{s s}(\rho)=1-\mathbb{E}\left[\frac{\sum_{k=0}^{2 N}(\sqrt{M}-1)^{k} \sum_{p=1}^{\left(\begin{array}{c}
2 N \\
k
\end{array}\right)} J_{k, p}}{M^{N}}\right],
$$

where $\mathbb{E}[\cdot]$ denotes expectation with respect to the channel coefficients distribution. Since each supersymbol consists of $N$ symbols, for the SEP it holds that

$$
P(\rho \mid \tilde{\mathbf{H}}) \geq \frac{P_{s s}(\rho \mid \tilde{\mathbf{H}})}{N} \quad \text { and } \quad P(\rho) \geq \frac{P_{s s}(\rho)}{N},
$$

where $P(\rho \mid \tilde{\mathbf{H}})$ and $P(\rho)$ are the corresponding error probabilities of the actual transmitted symbols.

\section{B. A Theoretical Limit for the SEP}

We define the parameter $W$, given by

$$
W=\frac{\sum_{i=1}^{N} \sum_{j=1}^{N} \gamma_{i, j}}{N},
$$


where $\gamma_{i, j}=\left|h_{i, j}\right|^{2}$ and $|\cdot|$ denotes the amplitude of its complex argument. We also define the $k$-dimensional spheres $\mathcal{B}_{k}$ with squared radius given by

$$
R_{k}^{2}=\frac{1}{\pi} \Gamma\left(\frac{k}{2}+1\right)^{\frac{2}{k}} W, \quad k=1,2, \ldots, 2 N,
$$

and the integrals $I_{k}$ given by

$$
I_{k}=\int_{\mathcal{B}_{k}} p\left(\mathbf{z}_{k}\right) \mathrm{d} \mathbf{z}_{k}=1-\frac{\Gamma\left(\frac{k}{2}, \frac{R_{k}^{2}}{2} \rho\right)}{\Gamma\left(\frac{k}{2}\right)}, \quad k=1,2, \ldots, 2 N,
$$

where $\Gamma(\cdot)$ and $\Gamma(\cdot, \cdot)$ are the Gamma function and the upper incomplete Gamma function, defined in [13, Eq. (8.310)] and [13, Eq. (8.350)], respectively. Finally we define $I_{0} \triangleq 1$.

Theorem 1: For a given channel realization $\tilde{\mathbf{H}}$, the SEP is lower-bounded by

$$
P_{l}(\rho \mid \tilde{\mathbf{H}})=\frac{M^{N}-\sum_{k=0}^{2 N}(\sqrt{M}-1)^{k}\left(\begin{array}{c}
2 N \\
k
\end{array}\right) I_{k}}{N M^{N}} .
$$

Proof: Since the volume of $\mathcal{V}_{\mathcal{S}_{k, p}}$ is the same as the volume of the corresponding fundamental parallelotope of the sublattice defined by $\mathcal{S}_{k, p}$ for a specific channel realization, as a consequence of Hadamard's inequality, it holds that

$$
\operatorname{vol}_{k}\left(\mathcal{V}_{\mathcal{S}_{k, p}}\right) \leq \prod_{i: \tilde{\mathbf{h}}_{i} \in \mathcal{S}_{k, p}}\left\|\tilde{\mathbf{h}}_{i}\right\|
$$

where $\operatorname{vol}_{k}(\cdot)$ denotes the $k$-dimensional volume, $\|\cdot\|$ denotes a vector's Euclidean norm and the equality holds only when the vectors $\tilde{\mathbf{h}}_{i}$ are orthogonal. Summing the squared values for all enumeration indexes, (17) yields

$$
\sum_{p=1}^{\left(\begin{array}{c}
2 N \\
k
\end{array}\right)} \operatorname{vol}_{k}^{2}\left(\mathcal{V}_{\mathcal{S}_{k, p}}\right) \leq \sum_{p=1}^{\left(\begin{array}{c}
2 N \\
k
\end{array}\right)} \prod_{i: \tilde{\mathbf{h}}_{i} \in \mathcal{S}_{k, p}}\left\|\tilde{\mathbf{h}}_{i}\right\|^{2},
$$

which can also be written as

$$
\begin{aligned}
& \sum_{p=1}^{\left(\begin{array}{l}
2 N \\
k
\end{array}\right)} \operatorname{vol}_{k}^{2}\left(\mathcal{V}_{\mathcal{S}_{k, p}}\right) \leq \\
& \sum_{\substack{b_{1}+b_{2}+\ldots+b_{2 N}=k \\
b_{1}, b_{2}, \ldots, b_{2 N} \in\{0,1\}}}\left(\left\|\tilde{\mathbf{h}}_{1}\right\|^{2}\right)^{b_{1}}\left(\left\|\tilde{\mathbf{h}}_{2}\right\|^{2}\right)^{b_{2}} \ldots\left(\left\|\tilde{\mathbf{h}}_{2 N}\right\|^{2}\right)^{b_{2 N}} .
\end{aligned}
$$

Using Maclaurin's Inequality [14, p.52], for $a_{1}, a_{2}, \ldots, a_{2 N} \in$ $\mathbb{R}$ and $0<k \leq 2 N$, we obtain

$$
\varrho_{2 N}^{\frac{1}{2 N}} \leq \varrho_{k}^{\frac{1}{k}} \leq \varrho_{1}
$$

where

$$
\varrho_{k}=\frac{\sum_{\substack{b_{1}+b_{2}+\ldots+b_{2 N}=k \\
b_{1}, b_{2}, \ldots, b_{2 N} \in\{0,1\}}} a_{1}^{b_{1}} a_{2}^{b_{2}} \cdots a_{2 N}^{b_{2 N}}}{\left(\begin{array}{c}
2 N \\
k
\end{array}\right)} .
$$

If we set $a_{i}=\left\|\tilde{\mathbf{h}}_{i}\right\|^{2}, i=1,2, \ldots, 2 N$, then $\varrho_{1}=\frac{\sum_{i=1}^{2 N}\left\|\tilde{\mathbf{h}}_{i}\right\|^{2}}{2 N}$. From (3), one can easily obtain

$$
\left\|\tilde{\mathbf{h}}_{j}\right\|^{2}=\left\|\tilde{\mathbf{h}}_{N+j}\right\|^{2}=\sum_{i=1}^{N}\left(\Re^{2}\left(h_{i, j}\right)+\Im^{2}\left(h_{i, j}\right)\right)=\sum_{i=1}^{N} \gamma_{i, j},
$$

for $j=1,2, \ldots, N$, where $h_{i, j}$ is the $(i, j)$ th element of the complex channel matrix $\mathbf{H}$. Therefore using (13), it is $\varrho_{1}=$ $\frac{2 \sum_{i=1}^{N} \sum_{j=1}^{N} \gamma_{i, j}}{2 N}=W$ and thus from (20) it holds that

$$
\begin{gathered}
\sum_{\substack{b_{1}+b_{2}+\ldots+b_{2 N}=k \\
b_{1}, b_{2}, \ldots, b_{2 N} \in\{0,1\}}}\left(\left\|\tilde{\mathbf{h}}_{1}\right\|^{2}\right)^{b_{1}}\left(\left\|\tilde{\mathbf{h}}_{2}\right\|^{2}\right)^{b_{2}} \cdots\left(\left\|\tilde{\mathbf{h}}_{2 N}\right\|^{2}\right)^{b_{2 N}} \\
\leq\left(\begin{array}{c}
2 N \\
k
\end{array}\right) W^{k}
\end{gathered}
$$

and combining (19) and (23) yields

$$
\sum_{p=1}^{\left(\begin{array}{c}
2 N \\
k
\end{array}\right)} \operatorname{vol}_{k}^{2}\left(\mathcal{V}_{\mathcal{S}_{k, p}}\right) \leq\left(\begin{array}{c}
2 N \\
k
\end{array}\right) W^{k}
$$

If $\mathcal{B}_{\mathcal{S}_{k, p}}$ is a $k$-dimensional sphere with radius $R_{\mathcal{S}_{k, p}}$ and volume $\operatorname{vol}_{k}\left(\mathcal{B}_{\mathcal{S}_{k, p}}\right)=\operatorname{vol}_{k}\left(\mathcal{V}_{\mathcal{S}_{k, p}}\right)=\frac{\pi^{\frac{k}{2}} R_{\mathcal{S}_{k, p}}^{k}}{\Gamma\left(\frac{k}{2}+1\right)}$, then

$$
\sum_{p=1}^{\left(\begin{array}{c}
2 N \\
k
\end{array}\right)} \frac{\pi^{k} R_{\mathcal{S}_{k, p}}^{2 k}}{\left(\Gamma\left(\frac{k}{2}+1\right)\right)^{2}} \leq\left(\begin{array}{c}
2 N \\
k
\end{array}\right) W^{k} .
$$

Taking into account (14) and substituting $W$, gives

$$
\sum_{p=1}^{\left(\begin{array}{c}
2 N \\
k
\end{array}\right)} R_{\mathcal{S}_{k, p}}^{2 k} \leq\left(\begin{array}{c}
2 N \\
k
\end{array}\right) R_{k}^{2 k}, \quad 0<k \leq 2 N .
$$

Now the function $f(x ; a, b)=\Gamma\left(\frac{a}{2}, b x^{\frac{1}{a}}\right)$ with $a, b \in \mathbb{R}^{+}$is convex in $(0, \infty)$. Indeed

$$
\frac{\mathrm{d} f}{\mathrm{~d} x}=-\left(b x^{\frac{1}{a}}\right)^{\frac{a}{2}-1} e^{-b x^{\frac{1}{a}}} \frac{\mathrm{d}\left(b x^{\frac{1}{a}}\right)}{\mathrm{d} x}=-\frac{b^{\frac{a}{2}} x^{-\frac{1}{2}} e^{-b x^{\frac{1}{a}}}}{a},
$$

and

$$
\frac{\mathrm{d}^{2} f}{\mathrm{~d} x^{2}}=\frac{b^{\frac{a}{2}} x^{-\frac{3}{2}} e^{-b x^{\frac{1}{a}}}}{2 a}\left(1+\frac{2 b}{a} x^{\frac{1}{a}}\right)>0 \forall x \in(0, \infty) .
$$

From Jensen's Inequality for convex functions [14], we have

$$
\sum_{i=1}^{K} \Gamma\left(\frac{a}{2}, b x_{i}^{\frac{1}{a}}\right) \geq K \Gamma\left(\frac{a}{2}, b\left(\frac{\sum_{i=1}^{K} x_{i}}{K}\right)^{\frac{1}{a}}\right) .
$$

For $a=k, b=\frac{\rho}{2}, K=\left(\begin{array}{c}2 N \\ k\end{array}\right)$ and $x_{i}=R_{\mathcal{S}_{k, p}}^{2 k}$, we get

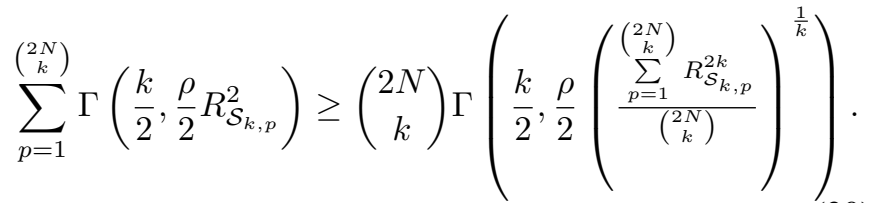

From (26) and since $f(x ; a, b)=\Gamma\left(\frac{a}{2}, b x^{\frac{1}{a}}\right)$ is a monotonically decreasing function with respect to $\mathrm{x}$,

$$
\Gamma\left(\frac{k}{2}, \frac{\rho}{2}\left(\frac{\sum_{p=1}^{\left(\begin{array}{c}
2 N \\
k
\end{array}\right)} R_{\mathcal{S}_{k, p}}^{2 k}}{\left(\begin{array}{c}
2 N \\
k
\end{array}\right)}\right)^{\frac{1}{k}}\right) \geq \Gamma\left(\frac{k}{2}, \frac{\rho}{2} R_{k}^{2}\right) .
$$


From (30) and (31), the following inequality is derived

$$
\sum_{p=1}^{\left(\begin{array}{c}
2 N \\
k
\end{array}\right)} \Gamma\left(\frac{k}{2}, \frac{\rho}{2} R_{\mathcal{S}_{k, p}}^{2}\right) \geq\left(\begin{array}{c}
2 N \\
k
\end{array}\right) \Gamma\left(\frac{k}{2}, \frac{\rho}{2} R_{k}^{2}\right)
$$

or equivalently

$$
\sum_{p=1}^{\left(\begin{array}{c}
2 N \\
k
\end{array}\right)}\left(1-\frac{\Gamma\left(\frac{k}{2}, \frac{\rho}{2} R_{\mathcal{S}_{k, p}}^{2}\right)}{\Gamma\left(\frac{k}{2}\right)}\right) \leq\left(\begin{array}{c}
2 N \\
k
\end{array}\right)\left(1-\frac{\Gamma\left(\frac{k}{2}, \frac{\rho}{2} R_{k}^{2}\right)}{\Gamma\left(\frac{k}{2}\right)}\right) .
$$

However, since $\operatorname{vol}_{k}\left(\mathcal{B}_{\mathcal{S}_{k, p}}\right)=\operatorname{vol}_{k}\left(\mathcal{V}_{\mathcal{S}_{k, p}}\right)$, it holds that [12]

$$
J_{k, p}=\int_{\mathcal{V}_{\mathcal{S}_{k, p}}} p\left(\mathbf{z}_{k}\right) \mathrm{d} \mathbf{z}_{k} \leq \int_{\mathcal{B}_{\mathcal{S}_{k, p}}} p\left(\mathbf{z}_{k}\right) \mathrm{d} \mathbf{z}_{k}=1-\frac{\Gamma\left(\frac{k}{2}, \frac{\rho}{2} R_{\mathcal{S}_{k, p}}^{2}\right)}{\Gamma\left(\frac{k}{2}\right)},
$$

Combining (33), (34) and taking into account (15) and the fact that $J_{0}=I_{0}=1$, we get

$$
\sum_{p=1}^{\left(\begin{array}{c}
2 N \\
k
\end{array}\right)} J_{k, p} \leq\left(\begin{array}{c}
2 N \\
k
\end{array}\right)\left(1-\frac{\Gamma\left(\frac{k}{2}, \frac{\rho}{2} R_{k}^{2}\right)}{\Gamma\left(\frac{k}{2}\right)}\right)=\left(\begin{array}{c}
2 N \\
k
\end{array}\right) I_{k},
$$

for any $0 \leq k \leq 2 N$. Using (9) (16) and (35) we finally have

$$
P_{s s}(\rho \mid \tilde{\mathbf{H}}) \geq 1-\frac{\sum_{k=0}^{2 N}(\sqrt{M}-1)^{k}\left(\begin{array}{c}
2 N \\
k
\end{array}\right) I_{k}}{M^{N}},
$$

and substituting (36) in (12) gives (16) and this concludes the proof.

Corollary 1: From Theorem 1, the SEP averaged over all channel realizations is lower-limited by

$$
P_{l}(\rho)=\frac{M^{N}-\sum_{k=0}^{2 N}(\sqrt{M}-1)^{k}\left(\begin{array}{c}
2 N \\
k
\end{array}\right) \mathbb{E}\left[I_{k}\right]}{N M^{N}} .
$$

\section{SOME PRACTICAL MIMO SYSTEMS}

The expression in (37) can be written in closed form for different fading distributions and correlation between the channel coefficients summed in (13). In the following we examine MIMO systems where the channel coefficient $h_{i, j}$ is assumed to be complex Gaussian and it can be written as $h_{i, j}=\left|h_{i, j}\right| e^{j \phi}$. The amplitude $\left|h_{i, j}\right|$ follows a Rayleigh distribution [15] with probability density function (pdf)

$$
p_{\left|h_{i, j}\right|}(x)=\frac{2 x}{\Omega} \exp \left(-\frac{x^{2}}{\Omega}\right),
$$

where $\Omega=\mathbb{E}\left[\left|h_{i, j}\right|^{2}\right]$ and $\mathbb{E}[\cdot]$ denotes expectation. Furthermore, if $\gamma_{i, j}=\left|h_{i, j}\right|^{2}$, then $\gamma_{i, j}$ follows an exponential distribution with pdf given by

$$
p_{\gamma_{i, j}}(x)=\frac{1}{\Omega} \exp \left(-\frac{x}{\Omega}\right) .
$$

Definition 1: We define the function

$$
f(\rho, N ; A, B, k)=\mathbb{E}\left[1-\frac{\Gamma\left(\frac{k}{2}, \frac{R_{k}^{2}}{2} \rho\right)}{\Gamma\left(\frac{k}{2}\right)}\right],
$$

where $0<k \leq 2 N$ and $R_{k}^{2}=A \sum_{i=1}^{B} \gamma_{i}$, We consider $\gamma_{i}$ as identically and independently distributed (i.i.d) random variables with pdf given in (39).

Lemma 1: The function in Definition 1 is given in closedform expression as

$$
f(\rho, N ; A, B, k)=1-\frac{1}{(A \Omega)^{B} \Gamma(B) \Gamma\left(\frac{k}{2}\right)} g\left(B, \frac{1}{A \Omega}, \frac{k}{2}, \frac{\rho}{2}\right),
$$

where

$$
\begin{aligned}
g(a, p, v, q)= & -\frac{q^{v} \Gamma(a+v)}{v p^{a+v}}{ }_{2} F_{1}\left(v, a+v ; v+1 ;-\frac{q}{p}\right) \\
& +\frac{\Gamma(v) \Gamma(a)}{p^{a}},
\end{aligned}
$$

and ${ }_{2} F_{1}(\cdot, \cdot ; \cdot ; \cdot)$ is the Gauss Hypergeometric function [13, Eq. (9.100), (9.14.2)].

Proof: The proof is given in Appendix A.

\section{A. The $N \times N$ Open-Loop Spatial Multiplexing Scheme}

We consider an $N \times N$ open-loop Spatial Multiplexing system [16], which can be directly represented by (1).

Proposition 1: The SEP of an $N \times N$ Spatial Multiplexing (SM) system is limited by

$$
P_{l}(\rho)=\frac{M^{N}+\sum_{k=1}^{2 N}(\sqrt{M}-1)^{k}\left(\begin{array}{c}
2 N \\
k
\end{array}\right) f\left(\rho, N ; A_{k}, B, k\right)}{N M^{N}} .
$$

with $A_{k}=\frac{1}{\pi N} \Gamma\left(\frac{k}{2}+1\right)^{\frac{2}{k}}$ for $0<k \leq 2 N$ and $B=N^{2}$.

Proof: In the SM case, all the channel coefficients of the channel matrix $\mathbf{H}$ are i.i.d random variables. Thus, from (13) we can derive the following parameters

$$
W=\frac{1}{N} \sum_{i=1}^{N^{2}} \gamma_{i}, \quad R_{k}^{2}=\frac{1}{\pi N} \Gamma\left(\frac{k}{2}+1\right)^{\frac{2}{k}} \sum_{i=1}^{N^{2}} \gamma_{i} .
$$

If $A_{k}=\frac{1}{\pi N} \Gamma\left(\frac{k}{2}+1\right)^{\frac{2}{k}}$ and $B=N^{2}$ then $R_{k}^{2}=A_{k} \sum_{i=1}^{B} \gamma_{i}$. Hence, using Lemma 1 and substituting in (37) we obtain (43) and this concludes the proof.

\section{B. An Orthogonal Code: The Alamouti $2 \times 1$ Scheme}

The Alamouti code [17] with 2 transmit and 1 receive antennas, is described by the well-known system of equations

$$
\left[\begin{array}{l}
y_{1} \\
y_{2}
\end{array}\right]=\left[\begin{array}{cc}
x_{1} & x_{2} \\
-x_{2}^{*} & x_{1}^{*}
\end{array}\right]\left[\begin{array}{l}
h_{1} \\
h_{2}
\end{array}\right]+\left[\begin{array}{l}
w_{1} \\
w_{2}
\end{array}\right]
$$

or equivalently (after signal processing at the receiver) by

$$
\left[\begin{array}{l}
y_{1} \\
y_{2}^{*}
\end{array}\right]=\left[\begin{array}{cc}
h_{1} & h_{2} \\
h_{2}^{*} & -h_{1}^{*}
\end{array}\right]\left[\begin{array}{l}
x_{1} \\
x_{2}
\end{array}\right]+\left[\begin{array}{c}
w_{1} \\
w_{2}^{*}
\end{array}\right]
$$

with the latter being expressed in the form of (1).

Proposition 2: The SEP of the $2 \times 1$ Alamouti code (AL) is limited by (43) with $N=2, A_{k}=\frac{1}{\pi} \Gamma\left(\frac{k}{2}+1\right)^{\frac{2}{k}}$ for $0<$ $k \leq 4$ and $B=N=2$.

Proof: In the $2 \times 1$ Alamouti code, only 2 out of 4 elements of the channel matrix $\mathbf{H}$ are i.i.d random variables, since the amplitude of any of the coefficients of the second 
row of the matrix $\mathbf{H}$ is the same as the amplitude of one of the coefficients of the first row. Thus, from (13) we get

$$
W=\frac{2 \sum_{i=1}^{2} \gamma_{i}}{2}=\sum_{i=1}^{2} \gamma_{i}, \quad R_{k}^{2}=\frac{1}{\pi} \Gamma\left(\frac{k}{2}+1\right)^{\frac{2}{k}} \sum_{i=1}^{2} \gamma_{i}
$$

If $A_{k}=\frac{1}{\pi} \Gamma\left(\frac{k}{2}+1\right)^{\frac{2}{k}}$ and $B=2$ then $R_{k}^{2}=A_{k} \sum_{i=1}^{B} \gamma_{i}$. From Lemma 1 and (37) for $N=2$ we conclude the proof.

\section{A Quasi-Orthogonal Code: The ABBA $4 \times 1$ Scheme}

The ABBA code [18] with 4 transmit and 1 receive antennas can be represented by the system of equations

$$
\left[\begin{array}{l}
y_{1} \\
y_{2} \\
y_{3} \\
y_{4}
\end{array}\right]=\left[\begin{array}{cccc}
x_{1} & x_{2} & x_{3} & x_{4} \\
-x_{2}^{*} & x_{1}^{*} & -x_{4}^{*} & x_{3}^{*} \\
x_{3} & x_{4} & x_{1} & x_{2} \\
-x_{4}^{*} & x_{3}^{*} & -x_{2}^{*} & x_{1}^{*}
\end{array}\right]\left[\begin{array}{l}
h_{1} \\
h_{2} \\
h_{3} \\
h_{4}
\end{array}\right]+\left[\begin{array}{l}
w_{1} \\
w_{2} \\
w_{3} \\
w_{4}
\end{array}\right],
$$

or equivalently by the following set of equations [11]

$$
\left[\begin{array}{c}
y_{1} \\
y_{2}^{*} \\
y_{3} \\
y_{4}^{*}
\end{array}\right]=\left[\begin{array}{cccc}
h_{1} & h_{2} & h_{3} & h_{4} \\
h_{2}^{*} & -h_{1}^{*} & h_{4}^{*} & -h_{3}^{*} \\
h_{3} & h_{4} & h_{1} & h_{2} \\
h_{4}^{*} & -h_{3}^{*} & h_{2}^{*} & -h_{1}^{*}
\end{array}\right]\left[\begin{array}{l}
x_{1} \\
x_{2} \\
x_{3} \\
x_{4}
\end{array}\right]+\left[\begin{array}{c}
w_{1} \\
w_{2}^{*} \\
w_{3} \\
w_{4}^{*}
\end{array}\right] .
$$

with the latter being expressed in the form of (1).

Proposition 3: The SEP of the $4 \times 1$ ABBA code is limited by (43) with $N=4, A_{k}=\frac{1}{\pi} \Gamma\left(\frac{k}{2}+1\right)^{\frac{2}{k}}$ for $0<k \leq 8$ and $B=N=4$.

Proof: In the $4 \times 1$ ABBA scheme, only 4 out of 16 elements of the channel matrix $\mathbf{H}$ are i.i.d random variables, since the amplitude of any of the coefficients in the rest of the rows of the matrix $\mathbf{H}$ is the same as the amplitude of one of the coefficients of the first row. Thus, from (13) it is

$$
W=\frac{4 \sum_{i=1}^{4} \gamma_{i}}{4}=\sum_{i=1}^{4} \gamma_{i}, \quad R_{k}^{2}=\frac{1}{\pi} \Gamma\left(\frac{k}{2}+1\right)^{\frac{2}{k}} \sum_{i=1}^{4} \gamma_{i} .
$$

If $A_{k}=\frac{1}{\pi} \Gamma\left(\frac{k}{2}+1\right)^{\frac{2}{k}}$ and $B=4$ then $R_{k}^{2}=A_{k} \sum_{i=1}^{B} \gamma_{i}$. Using Lemma 1 and (37) for $N=4$, we conclude the proof.

\section{Results AND Discussion}

In this section, we compare the performance limit in (37) with SEP simulation results for the three MIMO systems analyzed in Section IV. The target is to indicate the performance penalty for each of the selected schemes with respect to their analytical limits. We categorize the results according to the value of $N$, i.e. the size of the equivalent channel matrix $\mathbf{H}$ in (1). ${ }^{2}$ We consider two groups of systems; the first one $(N=2)$ includes the $2 \times 2 \mathrm{SM}$ and the $2 \times 1$ Alamouti, while the second $(N=4)$ comprises the $4 \times 4 \mathrm{SM}$ and the $4 \times 1$ ABBA. For the sake of convenience, we notate these designs as $\mathrm{SM}_{N=2}, \mathrm{AL}_{N=2}, \mathrm{SM}_{N=4}$, and $\mathrm{ABBA}_{N=4}$, respectively. For

\footnotetext{
${ }^{2}$ In the sense that the associated systems can be represented by an $N \times N$ complex system of equations.
}

all cases, we consider Rayleigh fading with average power $\Omega=1$, SQAM modulation format and ML decoding. Finally, all error probability curves are plotted versus the SNR per receive antenna $\left(\rho^{\prime}\right)$, which is related to the VNR $\rho$ defined in Section II according to $\rho^{\prime}=\alpha \rho$, where $\alpha=\Omega N(M-1) / 12$.

In Fig. 1, the simulation-based SEP curves of the $\mathrm{SM}_{N=2}$ and $\mathrm{AL}_{N=2}$ systems are plotted together with their limits derived in Section IV, for 4-QAM and 16-QAM. Apparently, the theoretical limit for the $\mathrm{AL}_{N=2}$, given in Proposition 2, is extremely tight to the actual ML performance for both spectral efficiencies (2 and $4 \mathrm{bps} / \mathrm{Hz}$ ). This is expected, since the Alamouti scheme is a full diversity system and the equivalent channel matrix is orthogonal. However, the limit which corresponds to the $\mathrm{SM}_{N=2}$ is far from the simulated ML performance. This happens because this limit bounds a nonexistent orthogonal scheme with all i.i.d channel coefficients.

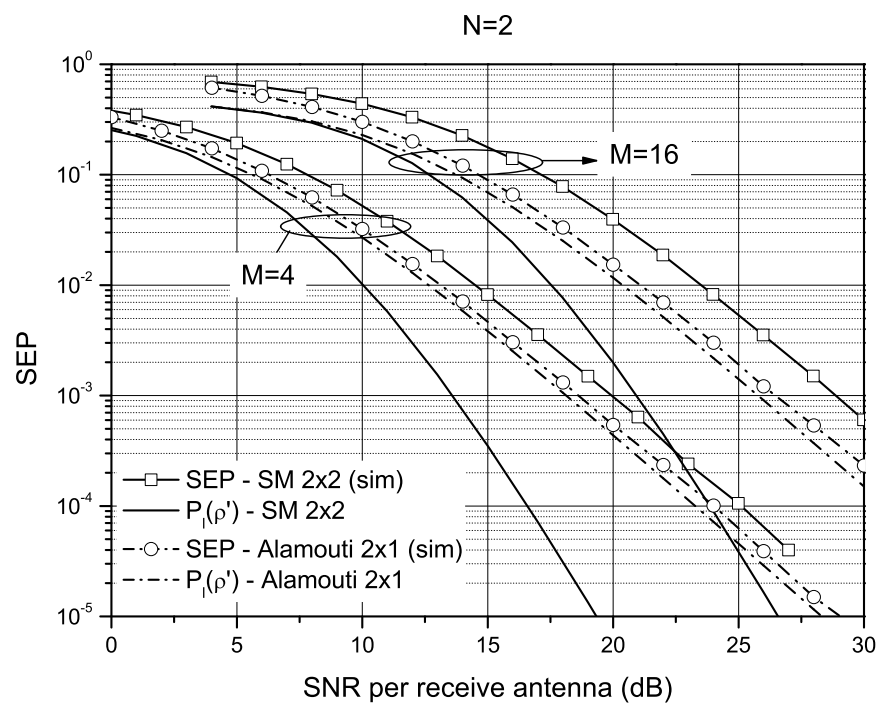

Fig. 1. SEP and $P_{l}\left(\rho^{\prime}\right)$ for the $2 \times 2$ open-loop SM case and the Alamouti $2 \times 1$ code for 4-QAM and 16-QAM (size of equivalent system $N=2$ ).

In Fig. 2, the ML performance simulation results and the corresponding theoretical limits of the $\mathrm{SM}_{N=4}$ and $\mathrm{ABBA}_{N=4}$ systems are illustrated. Again, the limit corresponding to the $\mathrm{SM}_{N=4}$ is far from its actual ML performance since it bounds an ideal $4 \times 4$ orthogonal system with all i.i.d channel coefficients, which though is non-existent. Nevertheless, for the $\mathrm{ABBA}_{N=4}$, the SEP curve gets closer to the corresponding limit, since its equivalent channel matrix offers half orthogonality due to the quasi-orthogonal structure of the ABBA code.

By taking a closer look at these results, it becomes evident that the performance limit in (37) can act as a theoretical benchmark for the efficient design of MIMO systems that can be written in the form of (1). This expression takes into account the correlation of the equivalent channel coefficients and it tightly bounds the performance of a full diversity system - ideal or existent - with the given channel correlation status. 


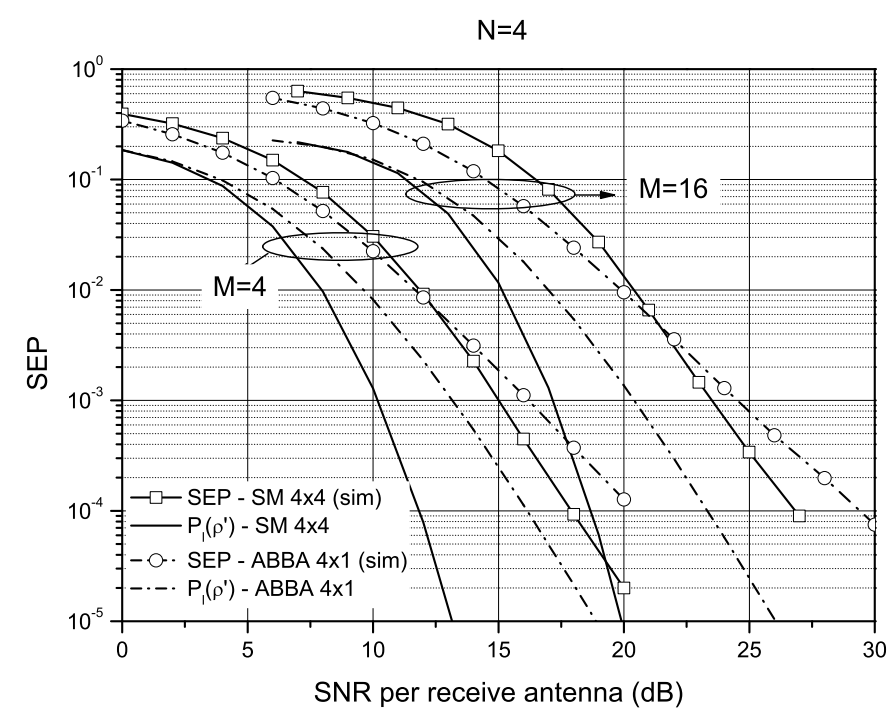

Fig. 2. SEP and $P_{l}\left(\rho^{\prime}\right)$ for the $4 \times 4$ open-loop SM case and the ABBA $4 \times 1$ code for 4-QAM and 16-QAM (size of equivalent system $N=4$ ).

\section{CONCLUSION}

In this work, elements from the theory of multidimensional constellations were employed to provide a solid framework for the lattice representation of general MIMO systems. With the aid of this geometrical interpretation, closed-form expressions for the performance limits of various multiple-antenna systems were derived and given in terms of symbol error probability under ML decoding. Simulations were conducted for three key MIMO systems with different multiplexing-diversity tradeoffs. The results reveal that designs exploiting in a better way the available degrees of freedom get closer to the derived theoretical limits, thus giving evidence of their performance optimality.

\section{APPENDix A \\ PROOF OF LEMMA 1}

We begin the proof by denoting $\mathcal{X}=\sum_{i=1}^{B} \gamma_{i}$, which is a sum of $B$ i.i.d exponential random variables and thus it is gamma distributed as [19]

$$
p_{\mathcal{X}}(x)=\frac{1}{\Omega^{B} \Gamma(B)} x^{B-1} \exp \left(-\frac{x}{\Omega}\right) .
$$

If (14) $R_{k}^{2}=A \mathcal{X}$ with $A>0$ then

$$
p_{R_{k}^{2}}(x)=\frac{1}{(A \Omega)^{B} \Gamma(B)} x^{B-1} \exp \left(-\frac{x}{A \Omega}\right) .
$$

Now

$$
\mathbb{E}\left[\Gamma\left(\frac{k}{2}, \frac{R_{k}^{2}}{2} \rho\right)\right]=\int_{0}^{\infty} \Gamma\left(\frac{k}{2}, \frac{\rho}{2} x\right) p_{R_{k}^{2}}(x) \mathrm{d} x .
$$

or equivalently

$\mathbb{E}\left[\Gamma\left(\frac{k}{2}, \frac{R_{k}^{2}}{2} \rho\right)\right]=\frac{(A \Omega)^{-B}}{\Gamma(B)} \int_{0}^{\infty} x^{B-1} \Gamma\left(\frac{k}{2}, \frac{\rho}{2} x\right) e^{-\frac{x}{A \Omega}} \mathrm{d} x$.
Using [20, Eq. (2.10.3.2)], (54) becomes

$$
\mathbb{E}\left[\Gamma\left(\frac{k}{2}, \frac{R_{k}^{2}}{2} \rho\right)\right]=\frac{(A \Omega)^{-B}}{\Gamma(B)} g\left(B, \frac{1}{A \Omega}, \frac{k}{2}, \frac{\rho}{2}\right),
$$

where $g(\cdot, \cdot, \cdot, \cdot)$ is given in (42). Using (55) in (40) yields (41) and this concludes the proof.

\section{REFERENCES}

[1] H. Bolcskei, D. Gesbert, C. Papadias, and A.-J. van der Veen, (Eds.) Space-time wireless systems: From array processing to MIMO communications. Cambridge University Press, 2006.

[2] C. Windpassinger, L. Lampe, R. F. H. Fischer, and T. Hehn, "A performance study of MIMO detectors," IEEE Trans. Wireless Commun., vol. 5, no. 8, pp. 2004-2008, Aug. 2006.

[3] K. Kuchi, "Limiting behavior of ZF/MMSE linear equalizers in wideband channels with frequency selective fading," IEEE Commun. Lett., vol. 16, no. 6, pp. 929-932, Jun. 2012.

[4] J. W. Choi, B. Shim, A. C. Singer, and N. I. Cho, "Low-complexity decoding via reduced dimension maximum-likelihood search," IEEE Trans. Signal Process., vol. 58, no. 3, pp. 1780-1793, Mar. 2010.

[5] P. Garg, R. K. Mallik, and H. M. Gupta, "Exact error performance of square orthogonal space-time block coding with channel estimation," IEEE Trans. Commun., vol. 54, no. 3, pp. 430-437, Mar. 2006.

[6] D. N. Dào and C. Tellambura, "Quasi-orthogonal STBC with minimum decoding complexity: Performance analysis, optimal signal transformations, and antenna selection diversity," IEEE Trans. Commun., vol. 56, no. 6, pp. 849-853, Jun. 2008.

[7] D. Senaratne, C. Tellambura, and H. A. Suraweera, "Performance analysis of MIMO channel inversion in Rayleigh fading," IEEE Trans. Vehic. Commun., vol. 61, no. 3, pp. 1188-1196, Mar. 2012.

[8] A. Nezampour and R. Schober, "Asymptotic analysis of space-time codes in generalized fading channels," IEEE Commun. Lett., vol. 13, no. 8, pp. 561-563, Aug. 2009.

[9] K. Kuchi and A. B. Ayyar, "Performance analysis of ML detection in MIMO systems with co-channel interference," IEEE Commun. Lett., vol. 15, no. 8, pp. 786-788, Aug. 2011.

[10] K. N. Pappi, N. D. Chatzidiamantis, and G. K. Karagiannidis, "A combinatorial geometrical approach to the error performance of multidimensional finite lattice constellations," in Proc. Wireless Communications and Networking Conference (WCNC) 2012, Paris, France, Apr. 2012.

[11] V. M. Kapinas and G. K. Karagiannidis, "On decoupling of quasiorthogonal space-time block codes based on inherent structure," in Proc. 16th IST Mobile and Wireless Communications Summit, Budapest, Hungary, Jul. 2007.

[12] A. G. Fàbregas and E. Viterbo, "Sphere lower bound for rotated lattice constellations in fading channels," IEEE Trans. Wireless Commun., vol. 7, no. 3, pp. 825-830, Mar. 2008.

[13] I. S. Gradshteyn and I. M. Ryzhik, Table of Integrals, Series, and Products, 7th ed. New York: Academic Press, 2007.

[14] G. H. Hardy, J. E. Littlewood, and G. Polya, Inequalities, 2nd ed. Cambridge, U.K.: Cambridge Univ. Press, 1952.

[15] M. K. Simon and M.-S. Alouini, Digital Communication over Fading Channels, 2nd ed. New York: Wiley, 2005.

[16] G. J. Foschini, "Layered space-time architecture for wireless communications in a fading environment when using multi-element antennas," Bell Labs Tech. J., pp. 41-59, 1996.

[17] S. M. Alamouti, "A simple transmit diversity technique for wireless communications," IEEE J. Select. Areas Commun., vol. 16, no. 8, pp. 1451-1458, Oct. 1998.

[18] O. Tirkkonen, A. Boariu, and A. Hottinen, "Minimal non-orthogonality rate 1 space-time block code for 3+ Tx antennas," in Proc. IEEE ISSSTA 2000, New Jersey, USA, Sep. 2000.

[19] A. Papoulis, S. U. Pillai, Probability, Random Variables and Stochastic Processes, 4th ed. McGraw-Hill, 2002.

[20] A. P. Prudnikov, Y. A. Brychkov, and O. I. Marichev, Integral and Series. Vol. 2: Special functions. Amsterdam: Gordon and Breach Science Publishers, 1986. 